\title{
The ability and utility of diffusion-weighted magnetic resonance imaging with different ' $b$ ' values in the differentiation of benign from malignant lung lesions
}

\author{
Şenol Fatih Elbir ${ }^{1 A, B, C, D, E}$, Mehmet Öztürk²A,B,C,D, Serap Doğan ${ }^{3 E, F, G}$ \\ 'Department of Radiology, Private Gözde Academy Hospital, Malatya, Turkey \\ 2Department of Radiology, Faculty of Medicine, Selcuk University, Konya, Turkey \\ ${ }^{3}$ Department of Radiology, Erciyes University Medical School, Kayseri, Turkey
}

\section{Abstract}

Purpose: To evaluate the ability and the utility of diffusion-weighted magnetic resonance imaging (MRI) with different ' $b$ ' values to visualise benign and malignant lung lesions, and to determine which 'b' value $\left(b=300,500\right.$, or $\left.1000 \mathrm{~s} / \mathrm{mm}^{2}\right)$ was most useful in differentiating benign from malignant lung lesions.

Material and methods: A total of 100 patients ( 28 women, 72 men; mean age $=57.19 \pm 13.44$ years; age range $=20-83$ years). Diffusion-weighted imaging (DWI) was obtained with ' $b$ ' values of 300,500 , and $1000 \mathrm{~s} / \mathrm{mm}^{2}$. The signal intensity of lesions on DWI images was analysed, and the apparent diffusion coefficient (ADC) values of the lesions were calculated. MRI was performed in all patients after having presented at our department for thoracic computed tomography for various reasons.

Results: A statistically significant difference in DWI signal scores was detected between benign and malignant lesions for all 'b' factors ( $p<0.0001$ for each). The sensitivity and specificity were $95 \%$ and $64 \%$, respectively, when a score of 3 for $\mathrm{b}=300 \mathrm{~s} / \mathrm{mm}^{2} ; 90 \%$ and $69 \%$, respectively, when a score of 3 for $\mathrm{b}=500 \mathrm{~s} / \mathrm{mm}^{2}$; and $84 \%$ and $74 \%$, respectively, when a score of 3 for $b=1000 \mathrm{~s} / \mathrm{mm}^{2}$. ADC values showed significant differences between benign and malignant lesions for all 'b' factors ( $p<0.0001$ for each).

Conclusions: Using 'b' values of 300, 500, and $1000 \mathrm{~s} / \mathrm{mm}^{2}$, DWI signal intensity scores and ADC values are effective methods for the differential diagnosis of malignant and benign pulmonary lesions.

Key words: b factor, diffusion-weighted magnetic resonance imaging, pulmonary lesions.

\section{Introduction}

Lung cancer is a leading cause of cancer death; hence, the characterisation of pulmonary nodules or masses by noninvasive methods is a major concern for radiologists and clinicians. Most pulmonary nodules share morphological similarities, and $25-39 \%$ of malignant nodules are assessed radiologically as benign [1]. Determining a radiological method that is accurate, as specific as possible, and minimally invasive in order to differentiate between benign and malignant lung lesions is considered an important diagnostic issue.

In the current algorithms for diagnostic guidelines for lung pathologies, radiography, computed tomography (CT), and positron emission tomography are commonly available. Motion artefacts in magnetic resonance imaging (MRI) of the blocker software, the use of multiple channels can be received now displays the desired quality in the thorax and fast, thanks to new technologies such as parallel imaging sequences. Diffusion-weighted imaging

Correspondence address:

Dr. Mehmet Öztürk, Department of Radiology, Faculty of Medicine, Selcuk University, Konya, Turkey, e-mail: drserapdogan@hotmail.com

Authors' contribution:

A Study design · B Data collection · C Statistical analysis · D Data interpretation · E Manuscript preparation · F Literature search · G Funds collection 
(DWI) is a noninvasive technique that reflects the random movement of water molecules (diffusion) in the tissues [2]. The sensitivity of DWI is regulated by different ' $b$ ' values. The ' $b$ ' value is a term defining the power of the diffusion sensitiser gradient [3]. Detection of more signal loss with higher ' $b$ ' values indicates that diffusion is affected not only by the movement of the water molecules but also by tissue perfusion $[4,5]$. DWI and the apparent diffusion coefficient (ADC) values provide important additional information beyond that of MRI. To the best of our knowledge, very few reports have described the application of DWI in the evaluation of pulmonary masses $[6,7]$.

The aim of this study was to evaluate the role of DW MRI with various ' $b$ ' values, to visualise pulmonary lesions and to determine which ' $b$ ' value $\left(300,500\right.$, or $\left.1000 \mathrm{~s} / \mathrm{mm}^{2}\right)$ was most useful in differentiating benign from malignant lung lesions.

\section{Material and methods}

\section{Patient selection}

This study was approved by our institutional review board, and informed consent was obtained from all patients. A total of 100 patients ( 28 women, 72 men; mean age, 57.19 \pm 13.44 years; age range, $20-83$ years) who had pulmonary nodules or masses that had a minimum nodule diameter of $1 \mathrm{~cm}$ were included in this study. MRI was performed in all patients after having presented at our department for thoracic CT for various reasons. In all 100 nodules/masses the final diagnoses were made histologically.

\section{Magnetic resonance imaging protocol}

All MRI examinations were performed using a commercially available 1.5-Tesla MRI unit (Philips Medical Systems, Best, Netherlands) with a linearly polarised body coil. All patients were examined in the supine position. For each patient, the following were used for the T1-weighted images: T1- and T2-weighted turbo spinecho (TSE) images were taken in the axial plane. TR/TE, $100 \mathrm{~ms} / 11 \mathrm{~ms}$; matrix, $256 \times 128$; section thickness, $6 \mathrm{~mm}$; section gap, $1 \mathrm{~mm}$; field of view, $36 \mathrm{~cm}$; flip angle, $70^{\circ}$; number of signals acquired, 1 . T2-weighted images were taken using respiratory gating. The following were used for the T2 images: TR/TE, $5000 \mathrm{~ms} / 80 \mathrm{~ms}$; matrix, $320 \times 224$ echo train length, 17 ; section thickness, $6 \mathrm{~mm}$; section gap, $1 \mathrm{~mm}$; field of view, $36 \mathrm{~cm}$; number of signals acquired, 2.

DWI were obtained with a single-shot echo-planar imaging sequence. DWI was performed with ' $b$ ' values of $0,300,500$, and $1000 \mathrm{~s} / \mathrm{mm}^{2}$. The following were used for DW images TR/TE, $5000 \mathrm{~ms} / 100 \mathrm{~ms}$; matrix, $128 \times 128$; section thickness, $8 \mathrm{~mm}$; section gap, $1 \mathrm{~mm}$; field of view, $360 \mathrm{~mm}$; R factor, 2; number of signals acquired, 4 . The data acquisition time for the DW images was 28 seconds.
All MR images were obtained during end-inspiration breath holding. Diffusion gradients were obtained in three planes ( $\mathrm{x}, \mathrm{y}$, and $\mathrm{z}$ ). ADC maps belonging to the isotropic images were created automatically by the device. The ADC values of the lesions were calculated in compliance with the ADC maps that were created in the second console of the MRI machine (View Forum R5.x, Philips Medical Systems).

\section{Image analysis}

All images and signal intensity data for the lesions on DWI with ' $b$ ' factors of 300,500 , and $1000 \mathrm{~s} / \mathrm{mm}^{2}$ were evaluated by two radiologists independently, who were blinded to the clinical data. Intensities were rated on a scale from 1 to 5 , in which: 1 - no signal intensity; 2 - signal intensity between 1 and 3; 3 - signal intensity similar to thoracic spinal cord intensity; 4 - over signal intensity than the spinal thoracic cord intensity; and 5 - much over signal intensity than the thoracic spinal cord intensity.

$\mathrm{ADC}$ measurements were performed by locating the region of interest (ROI) covering one-third of the lesions in accordance with the T2-weighted images by two radiologists. The ADC value was calculated automatically by locating three different ROIs in the lesion areas. The ROI was placed centrally, and the size of the ROI was kept as large as possible to cover at least two-thirds of the lesion while avoiding interference from the surrounding lung tissue, necrotic parts, and major blood vessels. The lowest of three values was used for statistical analysis. ADC maps of lung nodules were not available because of their susceptibility to artefacts. In this study, nodules with a diameter of $1 \mathrm{~cm}$ or larger were included. The diameter was calculated using the mean of the long- and short-axis diameters of nodules or masses on T1-weighted images.

\section{Statistical analysis}

The data were assessed using SPSS 15.0 and Sigma Stat 3.5 software. Comparison of categorical variables was performed by the exact method of Pearson's $\chi^{2}$ test. The Kolmogorov-Smirnov test was used to determine the normality of the distribution of the data. In variables showing normal and non-normal distributions in a comparison of the two groups, an independent two-sample $t$-test and the Mann-Whitney $U$-test were used, respectively. When the number of groups was greater than three, one-way variance analysis (one-way ANOVA) for the variables showing normal distributions, and Kruskal-Wallis analysis for variables showing non-normal distributions, were used. In multiple comparisons of those parameters that showed a difference in the one-way variance analysis, the Holm-Sidak test was used. To identify a suitable cut-off for the diagnostic tests, the receiver-operating characteristic (ROC) curve method was used. A $p$ value $<0.05$ was considered to indicate statistical significance. 


\section{Results}

The DWI signal intensity and ADC values of benign and malignant lesions in $\mathrm{b}=1000$ are summarised in Tables 1 and 2 .

The DWI signal scores of benign lesions were $2.85 \pm$ $1.47,2.70 \pm 1.30$, and $2.48 \pm 1.28$ with 'b' factors of 300 , 500 , and $1000 \mathrm{~s} / \mathrm{mm}^{2}$, respectively (Figure 1). The DWI signal scores of malignant lesions were $4.62 \pm 0.68,4.54$ \pm 0.72 , and $4.37 \pm 0.79$ with ' $b$ ' factors of 300,500 , and $1000 \mathrm{~s} / \mathrm{mm}^{2}$, respectively (Figure 2). However, a statistically significant difference in DWI signal scores was detected between benign and malignant lesions for all ' $b$ ' factors ( $p<0.0001$ for each). A signal decrease in DWI was observed in five malignant lesions: four adenocarcinomas and one squamous cell carcinoma. A signal increase in DWI was seen in 11 benign lesions: one Wegener's granulomatosis, four chronic active pus, one neurilemmoma, two organising pneumonia, one desquamative interstitial pneumonia, one extramedullary haematopoiesis, and one granulomatous lesion. We used the receiver operating characteristic (ROC) curve to evaluate the diagnostic capability of the five-point rank scale for differentiation between malignant and benign lesions. ROC analysis showed areas under the ROC curve of 0.825 for $b=300,0.866$ for $b=500$, and 0.869 for $b=1000 \mathrm{~s} / \mathrm{mm}^{2}$. ROC analysis showed $95 \%$ confidence intervals $(\mathrm{CI})$ of $0.738-0.893$ for $b=300,0.785-0.926$ for $b=500$, and $0.785-0.926$ for $\mathrm{b}=1000 \mathrm{~s} / \mathrm{mm}^{2}$. The area under the curve was highest for $\mathrm{b}=1000 \mathrm{~s} / \mathrm{mm}^{2}$. The sensitivity and specificity were, respectively, $95 \%$ and $64 \%$ for a score of 3 for $\mathrm{b}=300 \mathrm{~s} / \mathrm{mm}^{2}$, $90 \%$ and $69 \%$ for a score of 3 for $\mathrm{b}=500 \mathrm{~s} / \mathrm{mm}^{2}$, and $84 \%$ and $74 \%$ for a score of 3 for $b=1000 \mathrm{~s} / \mathrm{mm}^{2}$. However, the mean signal scores of DWI calculated with $b=300$, 500 , and $1000 \mathrm{~s} / \mathrm{mm}^{2}$ showed no significant differences.

Apparent diffusion coefficient values showed significant differences between benign and malignant lesions for all ' $b$ ' factors for benign lesions $(2.17 \pm 0.85,1.94 \pm 0.59$, and $\left.1.87 \pm 0.60 \mathrm{~mm}^{2} / \mathrm{s}\right)$ and for malignant lesions ( $0.88 \pm$ $0.41,0.95 \pm 0.38$, and $0.89 \pm 0.32 \mathrm{~mm}^{2} / \mathrm{s}$ ) with ' $b$ ' values of 300,500 , and $1000 \mathrm{~s} / \mathrm{mm}^{2}$, respectively $(p<0.0001$ for each). The mean ADC value was 1.69 in the three adenocarcinomas, 1.51 in one adenocarcinoma, and 1.58 in one squamous cell carcinoma. The mean ADC values of two plasmacytoma cases were 0.62 and 1.68 for a 'b' factor of $1000 \mathrm{~s} / \mathrm{mm}^{2}$, respectively. We used the receiver operating characteristic (ROC) curve to evaluate the diagnostic capability of the ADC values for differentiation between malignant and benign lesions. ROC analysis showed an area under the ROC curve of 0.898 for $b=300,0.897$ for $\mathrm{b}=500$, and 0.899 for $\mathrm{b}=1000 \mathrm{~s} / \mathrm{mm}^{2}$. ROC analysis showed 95\% CI of 0.823-0.949 for $b=300,0.821-0.948$ for $b=500$, and $0.823-0.950$ for $b=1000 \mathrm{~s} / \mathrm{mm}^{2}$. ROC analysis of $\mathrm{ADC}$ values revealed that the area under the curve for $b=1000 \mathrm{~s} / \mathrm{mm}^{2}$ was highest. The sensitivity and specificity were, respectively, $93 \%$ and $82 \%$ when the value
Table 1. Diffusion-weighted imaging (DWI) signal intensity and apparent diffusion coefficient (ADC) values of benign lesions in $b=1000 \mathrm{~s} / \mathrm{mm}^{2}$

\begin{tabular}{|c|c|c|}
\hline Lesion type & $\begin{array}{c}\text { DWI } \\
\left(b=1000 \mathrm{~s} / \mathrm{mm}^{2}\right)\end{array}$ & $\begin{array}{c}A D C \\
\left(b=1000 \mathrm{~s} / \mathrm{mm}^{2}\right)\end{array}$ \\
\hline $\begin{array}{l}\text { Granulomatous lesion } \\
(n=9)\end{array}$ & $1.44 \pm 0.44$ & $1.96 \pm 0.51$ \\
\hline $\begin{array}{l}\text { Chronic inflammation } \\
(n=8)\end{array}$ & $2.00 \pm 0.75$ & $1.96 \pm 0.30$ \\
\hline $\begin{array}{l}\text { Chronic active inflammation } \\
(n=6)\end{array}$ & $4.00 \pm 0.89$ & $1.35 \pm 0.63$ \\
\hline $\begin{array}{l}\text { Organizing pneumonia ( } \\
=5 \text { ) }\end{array}$ & $2.60 \pm 1.34$ & $1.88 \pm 0.53$ \\
\hline $\begin{array}{l}\text { Wegener's granulomatosis } \\
(n=2)\end{array}$ & $4.00 \pm 1.41$ & $1.38 \pm 1.04$ \\
\hline $\begin{array}{l}\text { Alveolar proteinosis } \\
(n=1)\end{array}$ & 3.00 & 1.86 \\
\hline $\begin{array}{l}\text { Benign thymoma (type A) } \\
(n=1)\end{array}$ & 2.00 & 1.71 \\
\hline $\begin{array}{l}\text { Desquamative interstitial } \\
\text { pneumonia }(n=1)\end{array}$ & 4.00 & 1.78 \\
\hline $\begin{array}{l}\text { Extramedullary } \\
\text { hematopoiesis }(n=1)\end{array}$ & 4.00 & 0.63 \\
\hline Fibrotic tissue $(n=1)$ & 1.00 & 3.04 \\
\hline $\begin{array}{l}\text { Interstitial pneumonia } \\
(n=1)\end{array}$ & 2.00 & 2.79 \\
\hline $\begin{array}{l}\text { Lymphocytic interstitial } \\
\text { pneumonia }(n=1)\end{array}$ & 2.00 & 1.78 \\
\hline Schwannoma $(n=1)$ & 5.00 & 2.27 \\
\hline Tuberculosis $(n=1)$ & 2.00 & 1.87 \\
\hline
\end{tabular}

Table 2. Diffusion-weighted imaging (DWI) signal intensity and apparent diffusion coefficient (ADC) values of malignant lesions in $b=1000 \mathrm{~s} / \mathrm{mm}^{2}$

\begin{tabular}{|l|c|c|}
\hline Lesion type & $\begin{array}{c}\text { DWI } \\
\left(\mathbf{b}=1000 \mathrm{~s} / \mathrm{mm}^{2}\right)\end{array}$ & $\begin{array}{c}\text { ADC } \\
\left(\mathbf{b}=1000 \mathrm{~s} / \mathrm{mm}^{2}\right)\end{array}$ \\
\hline Adenocarcinoma $(n=25)$ & $4.33 \pm 0.90$ & $0.96 \pm 0.41$ \\
\hline Squamous cell carcinoma $(n=19)$ & $4.46 \pm 0.66$ & $0.91 \pm 0.22$ \\
\hline Metastases $(n=5)$ & $4.40 \pm 0.89$ & $1.09 \pm 0.35$ \\
\hline Small cell carcinoma $(n=4)$ & $5.00 \pm 0.00$ & $0.57 \pm 0.12$ \\
\hline Large cell carcinoma $(n=2)$ & $5.00 \pm 0.00$ & $0.75 \pm 0.40$ \\
\hline Malignant thymoma $(n=2)$ & $5.00 \pm 0.00$ & $0.55 \pm 0.10$ \\
\hline Plasmacytoma $(n=2)$ & $3.00 \pm 0.00$ & $0.85 \pm 0.32$ \\
\hline Malignant lymphoma $(n=1)$ & 4.00 & 0.72 \\
\hline Mesothelioma $(n=1)$ & 5.00 & 0.92 \\
\hline
\end{tabular}

was 1.26 for $\mathrm{b}=300 \mathrm{~s} / \mathrm{mm}^{2}, 89 \%$ and $87 \%$ when the value was 1.19 for $b=500 \mathrm{~s} / \mathrm{mm}^{2}$, and $97 \%$ and $77 \%$ when the value was 1.49 for $b=1000 \mathrm{~s} / \mathrm{mm}^{2}$ (Figure 3). However, the mean $\mathrm{ADC}$ values calculated with $\mathrm{b}=300,500$, or $1000 \mathrm{~s} / \mathrm{mm}^{2}$ showed no statistically significant differences. 

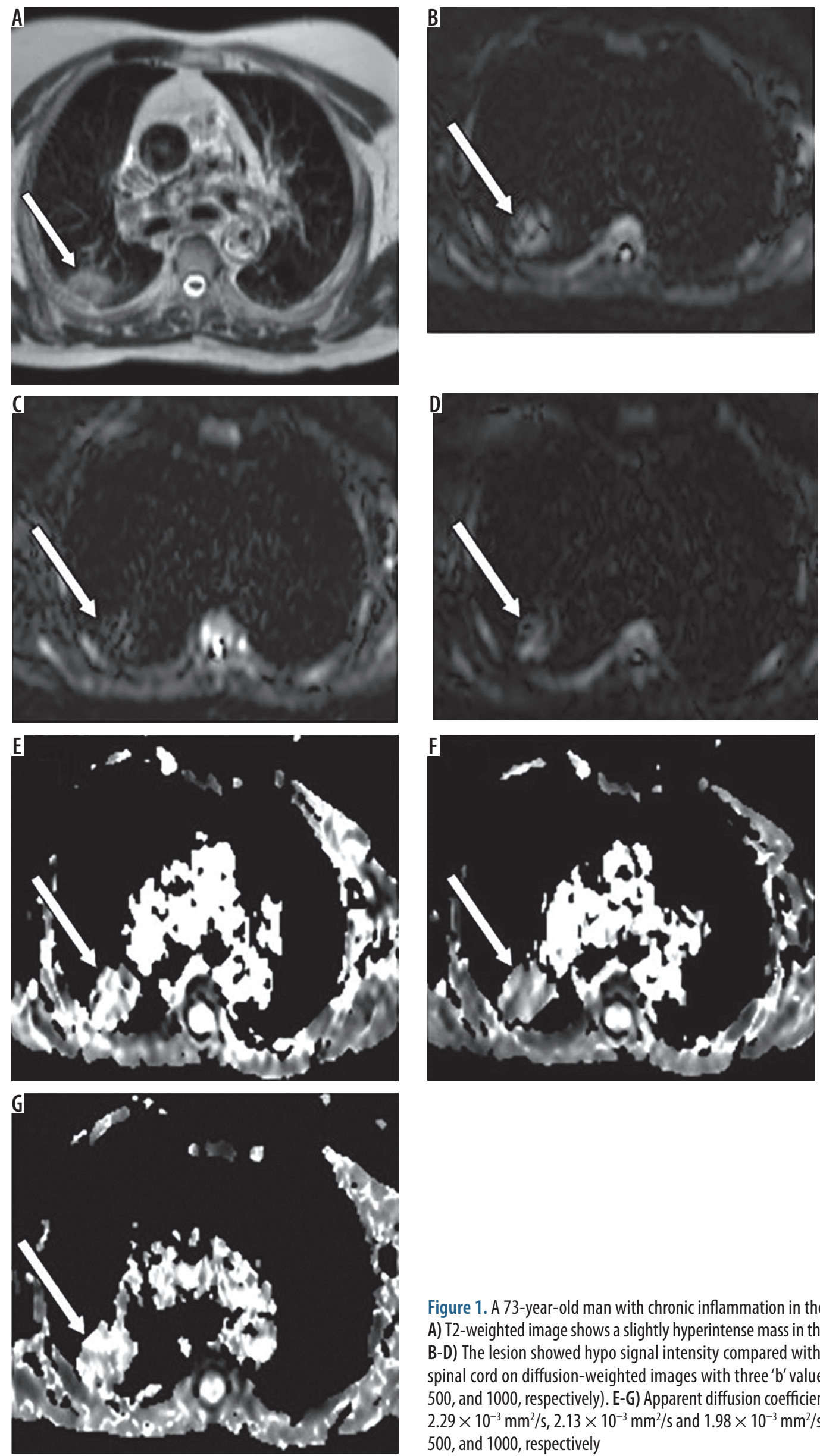

Figure 1. A 73-year-old man with chronic inflammation in the right lung. A) T2-weighted image shows a slightly hyperintense mass in the right lung. B-D) The lesion showed hypo signal intensity compared with the normal spinal cord on diffusion-weighted images with three ' $b$ ' values $(b=300$, 500 , and 1000 , respectively). E-G) Apparent diffusion coefficient values are $2.29 \times 10^{-3} \mathrm{~mm}^{2} / \mathrm{s}, 2.13 \times 10^{-3} \mathrm{~mm}^{2} / \mathrm{s}$ and $1.98 \times 10^{-3} \mathrm{~mm}^{2} / \mathrm{s}$ in $\mathrm{b}=300$, 500 , and 1000 , respectively 

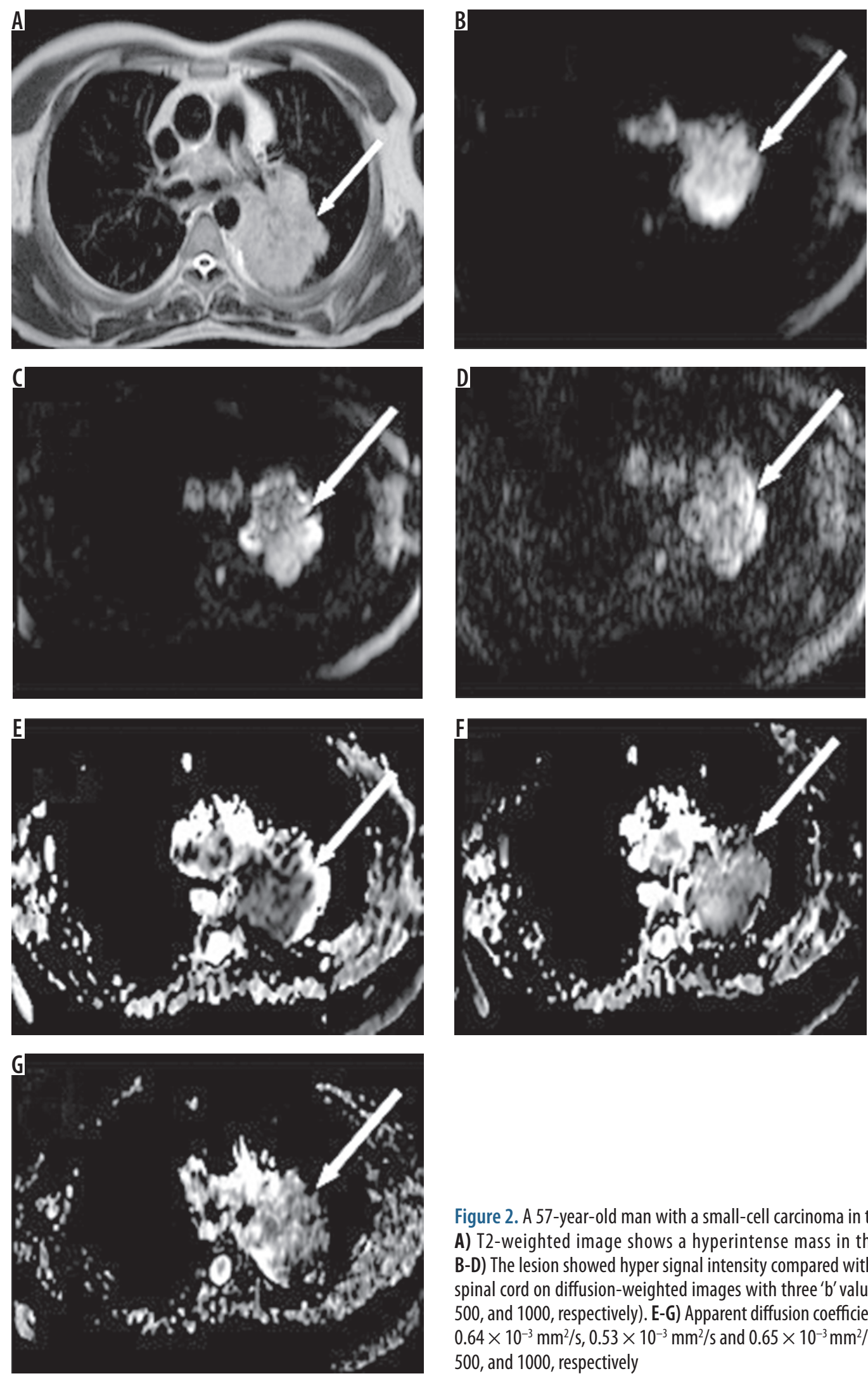

Figure 2. A 57-year-old man with a small-cell carcinoma in the left lung. A) T2-weighted image shows a hyperintense mass in the left lung. B-D) The lesion showed hyper signal intensity compared with the normal spinal cord on diffusion-weighted images with three ' $b$ ' values $(b=300$, 500 , and 1000, respectively). E-G) Apparent diffusion coefficient values are $0.64 \times 10^{-3} \mathrm{~mm}^{2} / \mathrm{s}, 0.53 \times 10^{-3} \mathrm{~mm}^{2} / \mathrm{s}$ and $0.65 \times 10^{-3} \mathrm{~mm}^{2} / \mathrm{s}$ in $\mathrm{b}=300$, 500 , and 1000 , respectively

\section{Discussion}

This study used different ' $b$ ' values for malignant-benign differentiation of lung masses and found two significant results.

The first is that the DWI signal score had high sensitivity and specificity for benign-malignant lung lesion differentiation. As a result, DWI is a useful tool for benign-malignant nodule differentiation. The second result is that a significant 


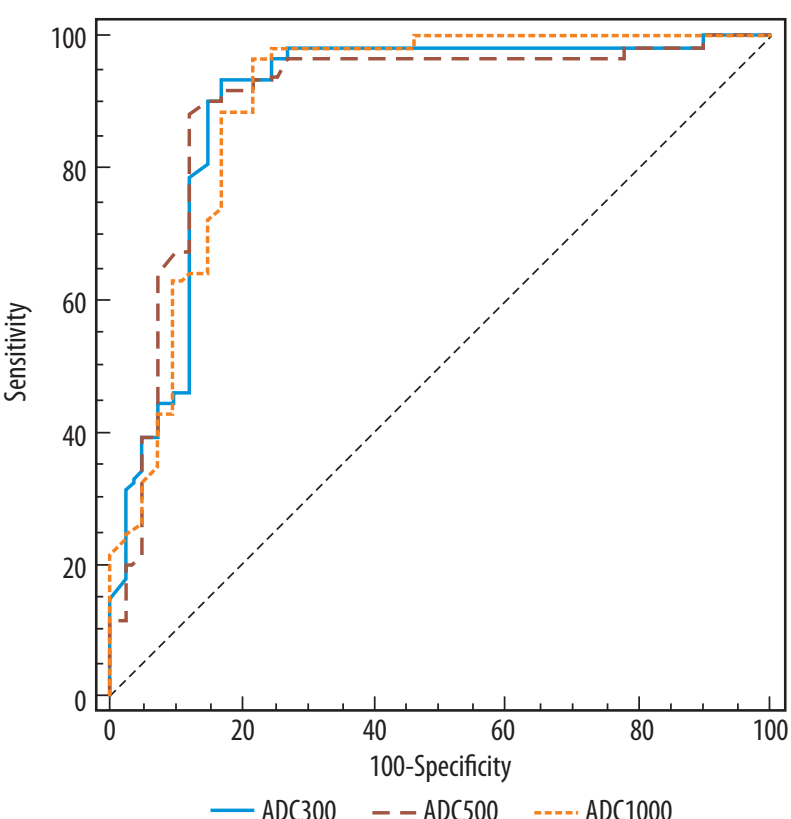

Figure 3. ROC analysis for critical apparent diffusion coefficient values in comparison of benign and malignant lesions in $b=300,500$, and 1000

difference was found for benign-malignant lung lesion differentiation using ADC. In other words, ADC can be used for benign-malignant lesion differentiation.

The $b=1000$ value contribution to DWI signal intensity for malignant-benign differentiation of lung masses with a threshold signal intensity score of 3 was used in two different studies. A study of 51 patients and 54 lesions by Satoh et al. [8] found $89 \%$ sensitivity, $61 \%$ specificity, and an area under the ROC curve of 0.87. A study carried out by Çakır et al. [7] included 46 patients with 48 lesions and found sensitivity of $93.3 \%$, specificity of $88.9 \%$, and an area under the ROC curve of 0.92 . In our study, we used three different ' $b$ ' values of 300,500 , and 1000, and with a signal score of 3 , there was no difference found in the sensitivity, specificity, or area under the ROC curve. However, the highest area under the ROC curve was found for $b=1000$ with an area of 0.87 , which is a finding that is similar to those of previous studies. Contrarily, in our study, the used moderate and low 'b' values had similar sensitivity and specificity rates to $\mathrm{b}=1000$. DWI is used with increasing frequency for lung pathologies, although there is still no standardised ' $b$ ' value $\left(\mathrm{s} / \mathrm{mm}^{2}\right)$ for thorax applications. Studies have generally used a relatively high ' $b$ ' value (1000). Additionally, there are research studies available on lung cancer diffusion characteristics that have obtained very good image quality using relatively low 'b' values $(68 / 577,0 / 500)$ [9-11]. However, to reduce $\mathrm{T} 2$ glare and intravoxel perfusion effects, high ' $\mathrm{b}$ ' values should be chosen. High 'b' values reduce SNR and reveal anatomic disruption.

Histologically, well-differentiated adenocarcinoma grow without disrupting the lung architecture and are sourced in the normal cycle of cylindrical cells in the airway walls before alveolus. Poorly differentiated adenocarcinoma and other lung tumours develop outside of the normal cycle of cells and form large expansive solid masses compressing their surroundings. As a result, the cellularity of well-differentiated tumours is lower than that of poorly differentiated adenocarcinoma and other lung tumours [9]. In our study, diffusion restriction was not observed in four adenocarcinoma cases or one squamous cell carcinoma case, with ADC values higher than in malignant masses. This situation is linked to the cases demonstrating well-differentiated tumours. Extramedullary plasmacytoma has the best prognosis among the plasma cell neoplasms. However, $10-40 \%$ of cases progress to develop multiple myeloma [12]. In our study, in neither of the two plasmacytoma cases was diffusion restricting. This is linked to the cellularity of plasmacytoma being low and well-differentiated. It is difficult to interpret non-solid neoplasms and active inflammatory pulmonary nodules using the DWI signal intensities. Differences in signal intensity reflect histopathological differences with malignant tumours that are generally hypercellular with large cells. Despite the fact that lung abscesses and active tuberculosis are benign pulmonary nodules, they contain very few inflammatory cells, and, as a result, high signal intensity may be observed [8]. In our study, DWI signal increase was observed for 11 benign lesions. Of these, one case had a schwannoma. This is linked to the clear hyperintensity of schwannoma on T2 due to the T2 effect. The signal increase for the other 10 lesions (four chronic active infection cases, two OP cases, one Wegener's granulomatosis, one desquamative interstitial pneumonia, one extramedullary haematopoiesis, and one granulomatous lesion) is linked to greater cellularity.

In a study of 18 benign and 30 malignant lesions using the $\mathrm{b}=1000$ value, Çakır et al. [7] found that the ADC value for benign lesions was $2.423 \pm 1.299 \times 10^{-3} \mathrm{~mm}^{2} / \mathrm{s}$, and for malignant lesions it was $1.185 \pm 0.236 \times 10^{-3} \mathrm{~mm}^{2} / \mathrm{s}$. In a study using the $b=500$ value for 12 benign and 54 malignant lesions, Liu et al. [10] found that the ADC value for benign lesions was $1.64 \pm 0.41 \times 10^{-3} \mathrm{~mm}^{2} / \mathrm{s}$, and for malignant lesions it was $1.25 \pm 0.32 \times 10^{-3} \mathrm{~mm}^{2} / \mathrm{s}$. In our study, the ADC values for benign lesions using $b=300$, $\mathrm{b}=500$, and $\mathrm{b}=1000$ were $2.17 \pm 0.85,1.94 \pm 0.59$, and $1.87 \pm 0.60$, respectively. The ADC values for malignant lesions were $0.88 \pm 0.41,0.95 \pm 0.38$, and $0.89 \pm 0.32$ for $\mathrm{b}=300, \mathrm{~b}=500$, and $\mathrm{b}=1000$, respectively. For both groups in which $\mathrm{ADC}$ values were measured $\mathrm{at} b=300, \mathrm{~b}=500$, and $b=1000$, we found a statistical difference that was similar to the findings of previous studies $(p<0.05)$. Çakır et al. [7] found $87 \%$ sensitivity and $89 \%$ specificity for the ADC threshold value of 1.5 using a ROC analysis with $b=1000$. Liu et al. [10] found $83 \%$ sensitivity and $74 \%$ specificity for a threshold value of 1.4 with $b=500$. The results of ADC mapping from ROC analysis in our study found that the sensitivity and specificity values for the $b=300$ threshold of 1.26 were $93 \%$ and $82 \%, b=500$ threshold 1.19 were $89 \%$ and $87 \%$, and $b=1000$ threshold 1.49 were $97 \%$ and $77 \%$, respectively. These values are in 
accordance with the literature, with the highest area under the ROC curve for $b=1000$. However, low ' $b$ ' values (500) have been used in the differentiation of post-obstructive atelectasis with lung cancer [13]. When low 'b' values are used, it has been reported that ADC values are found to be higher. In a study using low ' $b$ ' values in a phantom application, Matoba et al. obtained diffusion imaging in acetone and distilled water and measured the ADC values. Because their identified values were higher than presented in the literature for defined values for acetone and distilled water, the researchers proposed that this may have been due to the use of a low ' $b$ ' value because the tissue perfusion and T2 time directly affect ADC values. Thus, increasing the ' $b$ ' value reduces the $\mathrm{T} 2$ effect on imaging. In contrast, very high $(<1200)$ 'b' values are suggested to cause significant reduction in image quality due to shortened T2 time $[9,11]$.
One of our study's limitations is that it did not include lesions that were smaller than $1 \mathrm{~cm}$ in diameter. Such small lesions were not included in consideration of susceptibility artefacts due to the fact that air in the lungs and movement artefacts in lower zone and paracardiac localised lesions cause incorrect ADC measurements.

In conclusion, both DWI signal intensity scores and $\mathrm{ADC}$ values may be useful for the differentiation of benign and malignant lung lesions at all 'b' values. However, the value of DW-MRI in differentiating between pathological types of benign and malignant lesions needs further study.

\section{Conflict of interest}

The authors report no conflict of interest.

\section{References}

1. Gurney JW. Determining the likelihood of malignancy in solitary pulmonary nodules with Bayesian analysis. Part I. Theory. Radiology 1993; 186: 405-413.

2. Le Bihan D, Turner R, Douek P, Patronas N. Diffusion MR imaging: clinical applications. Am J Roentgenol 1992; 159: 591-599.

3. Naganava S, Kawai H, Fukatsu H. Diffusion-weighted imaging of the liver: technical challenges and prospects for the future. Magn Reson Med Sci 2005; 4: 175-186.

4. Taouli B, Vilgrain V, Dumont E, et al. Evaluation of liver diffusion isotropy and characterization of focal hepatic lesion with two singleshot echoplannar MR imaging sequences: prospective study in 66 patients. Radiology 2003; 226: 71-78.

5. Le Bihan, Breton E, Lallemand D, et al. MR imaging of intravoxel incoherent motions: application to diffusion and perfusion in neurologic disorders. Radiology 1986; 161: 401-407.

6. Wan Q, Deng YS, Zhou JX, et al. Intravoxel incoherent motion diffusion-weighted MR imaging in assessing and characterizing solitary pulmonary lesions. Sci Rep 2017; 7: 43257.

7. Çakır Ç, Gençhellaç H, Temizöz O, et al. Diffusion weighted magnetic resonance imaging for the characterization of solitary pulmonary lesions. Balkan Med J 2015; 32: 403-409.
8. Satoh S, Kitazume Y, Ohdama S, et al. Can malignant and benign pulmonary nodules be differentiated with diffusion weighted MRI? AJR Am J Roentgenol 2008; 191: 464-470.

9. Matoba M, Tonami H, Kondou T, et al. Lung carcinoma: diffusionweighted mr imaging preliminary evaluation with apparent diffusion coefficient. Radiology 2007; 243: 570-577.

10. Liu H, Liu Y, Yu T, Ye N. Usefulness of diffusion-weighted MR imaging in the evaluation of pulmonary lesions. Eur Radiol 2010; 20: 807-815.

11. Uto T, Takehara Y, Nakamura Y, et al. Higher sensitivity and specificity for diffusion weighted imaging of malignant lung lesions without apparent diffusion coefficient quantification. Radiology 2009; 252: 247-254.

12. Holland J, Trenkner DA, Wasserman TH, Fineberg B. Plasmacytoma. Treatment results and conversion to myeloma. Cancer 1992; 69: 1513-1517.

13. Qi LP, Zhang XP, Tang L, et al. Using diffusion weighted MR imaging for tumor detection in the collapsed lung: a preliminary study. Eur Radiology 2009; 19: 333-341. 